\title{
Alias-Free Interpolation
}

\author{
C.V. Jiji, Prakash Neethu, and Subhasis Chaudhuri \\ Indian Institute of Technology Bombay, Mumbai, India \\ $\{j i j i$, neethu, sc\}@ee.iitb.ac.in
}

\begin{abstract}
In this paper we study the possibility of removing aliasing in a scene from a single observation by designing an alias-free upsampling scheme. We generate the unknown high frequency components of the given partially aliased (low resolution) image by minimizing the total variation of the interpolant subject to the constraint that part of unaliased spectral components in the low resolution observation are known precisely and under the assumption of sparsity in the data. This provides a mathematical basis for exact reproduction of high frequency components with probability approaching one, from their aliased observation. The primary application of the given approach would be in super-resolution imaging.
\end{abstract}

\section{Introduction}

Images with high spatial resolution are always a necessity in computer vision applications. Resolution enhancement using interpolation techniques is of limited application because of the aliasing present in the low resolution (LR) image. Hence researchers have been working in the field of super-resolution (SR) where a high-resolution (HR) image is reconstructed using one or more LR observations. In general, super-resolution involves deblurring, denoising and alias-removal. There are, in general, two classes of super-resolution techniques: reconstructionbased and learning-based [1]. In reconstruction-based SR techniques several LR images are used to reconstruct the super-resolved image. In learning-based methods proposed in the literature, one or more LR observations are used, but they make use of a database of several HR images to estimate the HR image corresponding to the given LR image.

All existing papers claim that they have been able to generate additional high frequency components through the use of multiple exposures or learning from the database. But there has been no mathematical proof or studies to show that the generated high frequency components are, indeed, the correct ones! For example, even a bilinear interpolation will generate (spurious) high frequency components. Unlike all previous work, we provide a mathematical basis based on which the correctness of the generated high frequency components can be established. In this paper we study only one specific aspect of SR, the alias removal part, at an exact theoretical level. We deal with a very specific caseonly a single LR observation, no multiple view collation and no learning from a database. In effect, we show how much additional information can be extracted from a single observation through alias removal alone. 
The super-resolution idea was first proposed by Tsai and Huang [2]. Their frequency domain approach reconstructs an HR image from a sequence of several LR undersampled images by exploiting the relationship between the continuous and the discrete Fourier transforms of the undersampled frames. A different approach to the super-resolution restoration problem was suggested by Irani et al. 3] based on the iterative back projection method. A maximum aposteriori (MAP) estimator with Huber-Markov random field prior is described by Schultz and Stevenson in 4. Elad and Feuer [5] proposed a unified methodology for super-resolution restoration from several geometrically warped, blurred, noisy and down-sampled images by combining maximum likelihood, MAP and projection onto convex sets approaches. Nguyen et al. proposed circulant block preconditioners to accelerate the conjugate gradient descent method while solving the Tikhonov-regularized super-resolution problem [6].

In all the above methods, the quality of the super-resolved image is measured either by means of visual inspection or using a PSNR check. It can be easily shown that the PSNR measure is heavily biased towards the lower part of the spectrum due to the fact that most of the energy is contained in this region. Hence the PSNR may not be a good measure to evaluate the performance of an SR scheme. The issue that the reconstructed components are really the high frequency components has not really been investigated so far. Our work in this paper is a study in this direction. In [7], Lin and Shum determine the quantitative limits of reconstruction-based super-resolution algorithms and obtain the upsampling limits from the conditioning analysis of the coefficient matrix. But it is restricted to a perturbation analysis and not on spectral resolvability. Shahram and Milanfar in 8] study how far beyond the classical Rayleigh limit of resolution one can reach at a given signal to noise ratio using statistical analysis. Here the authors do not study the system performance in the presence of aliasing.

Rajan et al. have analyzed the possibility of alias-free upsampling of images in 9] through the use of a generalized interpolation. They have shown the conditions under which such an interpolation is possible. However, it requires several observations and the knowledge of a non-linear transform to achieve this. We study the issue of alias-free interpolation at a more fundamental level and restrict ourselves to using a single observation. Our work is motivated by the work of Candes et al. [10] where the authors address the problem of exact signal reconstruction from incomplete frequency information. We build on the theorem developed by them to derive a method for exact removal of aliasing while interpolating an image.

The reminder of the paper is organized as follows. We discuss the LR image formation process in section 2. We also define the problem here. A relevant theorem which we make use of in solving the problem is stated in 3. Some useful corollaries are also given. Section 4 explains our alias-free interpolation technique. In section 5 we discuss the computational scheme to solve the problem. We present experimental results on different types of images in section 6, and the paper concludes in section 7 . 


\section{$2 \quad$ Aliasing in LR Image}

It is assumed that the observed low resolution image is produced from a single high resolution image under the following generative model. Let $\bar{z}$ represent the lexicographically ordered high resolution image of $K^{2} \times 1$ pixels. If $\bar{g}$ is the $N^{2} \times 1$ lexicographically ordered vector containing pixels from the low resolution observation, then it can be modeled as

$$
\bar{g}=D A \bar{z}
$$

where $D$ is the decimation matrix, size of which depends on the decimation factor and $A$ is the blur matrix, assumed to be an identity matrix in this paper for the specific task of studying the alias-removal property.

The LR image formed through the above process will, in general, be aliased. The aliasing mechanism is illustrated in Figure 1. The spectrum of a continuoustime 1-D signal $x_{c}(t)$ band limited to $B$ is shown in Figure 1(a). The spectrum of the sampled signal $x(n)$ sampled at a rate $F<2 B$ is shown in Figure 1(b). Of course the spectrum will be aliased since the signal is sampled at a rate less than the Nyquist rate. The resultant aliased spectrum of the sampled signal is shown in Figure 1)(c). As can be noted from Figure 1(c) the portion of the spectrum $F-B \leq \omega \leq B$ will be aliased and the rest will be alias-free. A similar form of aliasing takes place in low resolution images unless the blur matrix $A$ in equation (11) is quite severe. The knowledge about the portion of the spectrum $0 \leq \omega \leq F-B$ will be used as a constraint, as these components are free from aliasing, in the proposed method to recover the high frequency components.

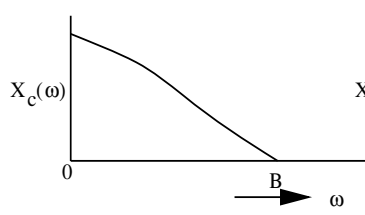

(a)

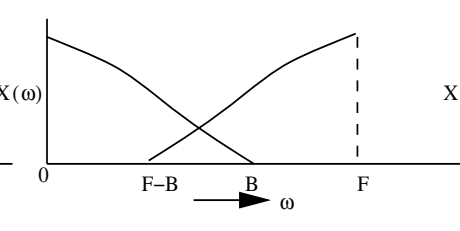

(b)

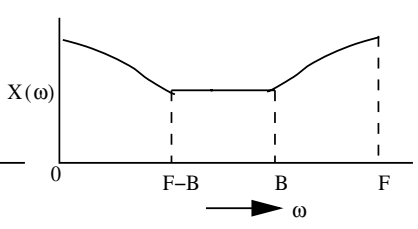

(c)

Fig. 1. Illustration of the aliasing process: (a) Spectrum of a continuous-time signal $x_{c}(t),(\mathrm{b})$ components of the spectrum of the sampled signal $x(n)$, and (c) resultant spectrum of $x(n)$

Having explained the aliasing process, we now define our problem in terms of alias-free interpolation: Given an LR image $g(x, y)$ of size $N \times N$ whose spectrum is partially aliased, generate an interpolated image $z(x, y)$ of size $2 N \times 2 N$ which is completely alias-free under the assumption that the image consists of piece-wise constant intensity regions. The significance of the assumption will be explained in the next section. 


\section{A Relevant Theorem by Candes et al.}

Theorem 1: Consider a discrete-time 1-D signal $f \in C^{N}$ and a randomly chosen set of frequencies $\Omega$ of mean size $\tau N, 0<\tau<1$. Then for each $\zeta>0$, suppose that $f$ obeys

$$
\#\{n, f(n) \neq 0\} \leq \alpha(\zeta) \cdot(\log N)^{-1} \cdot \# \Omega,
$$

then with probability at least $1-O\left(N^{-\zeta}\right), f$ can be reconstructed exactly as the solution to the $l_{1}$ minimization problem

$$
\min _{h} \sum_{n=0}^{N-1}|h(n)| \text { s.t. } H(\omega)=F(\omega) \forall \omega \in \Omega
$$

where $H(\omega)$ and $F(\omega)$ are the discrete Fourier transforms of $h(n)$ and $f(n)$ respectively and \# refers to the count.

Here $\zeta$ is an accuracy parameter in the term $O\left(N^{-\zeta}\right)$ and $\alpha(\zeta)$ has been shown to be equal to $(1+o(1)) /(29.6(\zeta+1))$ under certain conditions in 10 . In a simple language it means that as one selects more spectral components compared to the number of non-zero elements in $f(n)$, one is likely to recover the true function $f(n)$ with a higher accuracy. Proof of the theorem can be found in [10]. This is typically known as data sparsity problem [1], one such common example of which is inpainting 12 where one is required to reconstruct the missing data. An interesting reference to this work is by Chan et al. [13] where the authors investigate the reverse problem, i.e., how much loss in data can be tolerated for a faithful reconstruction of a signal as opposed to what Candes et al. [10] has studied.

According to the theorem the discrete-time signal $f$ can be reconstructed from its partial frequency samples as the solution to the constrained $l_{1}$ optimization problem as stated above. It may be noted that the reconstruction is possible if the signal consists of a limited number of spikes (Kronecker delta) only. However, most of the input signals or images do not satisfy the above condition. If we consider this function to be a derivative (forward difference in the discrete case) of the function $f(n)$, then the corresponding $l_{1}$ minimization should be performed on the derivative of $h(n)$, or in other words, one has to minimize the total variation (TV). This leads to the following corollary.

Corollary 1: A piecewise constant object can be reconstructed from incomplete frequency samples provided the number of discontinuities satisfy the above condition 2, as the solution to the minimization problem

$$
\min _{h} \sum_{n=0}^{N-1}|h(n)-h(n-1)| \text { s.t. } H(\omega)=F(\omega) \forall \omega \in \Omega
$$

Corollary 2: If $f(x, y)$ is a two-dimensional object, it can be reconstructed from its incomplete frequency samples as the solution to the minimization problem

$$
\min _{h} \sum \sum\left(\left|h_{x}\right|+\left|h_{y}\right|\right) \text { s.t. } H(\omega)=F(\omega) \forall \omega \in \Omega
$$


where $h_{x}(x, y)=h(x, y)-h(x-1, y)$ and $h_{y}(x, y)=h(x, y)-h(x, y-1)$. This is similar to minimizing the total variation norm of $h(x, y)$. But this is not rotationally symmetric.

It may be noted that the same solution was proposed in 1981 by Levy and Fullagar [14] in connection with the reconstruction of geophysical data. Also see the reference [15] for a similar work. Candes et al. have provided a theoretical footing of the existing solution. It may be noted that total variation-based image interpolation methods are also proposed in [16] and [17]. But the authors do not specifically address the issue of alias removal. For a detailed review of TV, readers are referred to $[18$.

\section{Alias-Free Interpolation}

The problem addressed in [10] is a restoration problem where the discrete-time signal is reconstructed from its incomplete Fourier samples such as in computed tomography. However, they do not consider the effect of aliasing on the sampled data. But our problem is a signal interpolation one, where only one LR observation $g$ is available, which is the decimated version of the unknown HR image $z$ as explained in section 2, Of course, $g$ will be aliased. We wish to remove this aliasing completely while interpolating the image assuming the aliasing to be only partial. It may be noted that without the interpolation (use of a denser grid to represent the data), one cannot recover the aliased components. To apply the above theorem to our problem, a partial knowledge about $Z(\omega)$ should be available. We now explain how a partial knowledge of $Z(\omega)$ can be obtained from the given observation $G(\omega)$. Our alias-free interpolation procedure is illustrated in Figure 2 with respect to a given 1-D LR sequence $g(n)$ of length $N$. Note that unlike in theorem 1 , we are dealing with real valued function $g(n)$ and hence the spectrum is always conjugate symmetric and one has to consider only one half of the spectral components. Figure 2(a) shows the partially aliased spectrum of the LR sequence $g(n)$ of length $N$. We assume that $G(\omega)$ in $0 \leq \omega \leq M$ is free from aliasing and the remaining portion is aliased. This corresponds to the assumption that the continuous signal $g_{c}(t)$ is band limited to the normalized frequency $(1-M / N)$, where $0 \leq M \leq N / 2$. The smaller the value of $M$, the larger is the amount of aliasing. Figure 2(b) shows the spectrum of the HR sequence $z(n)$ of

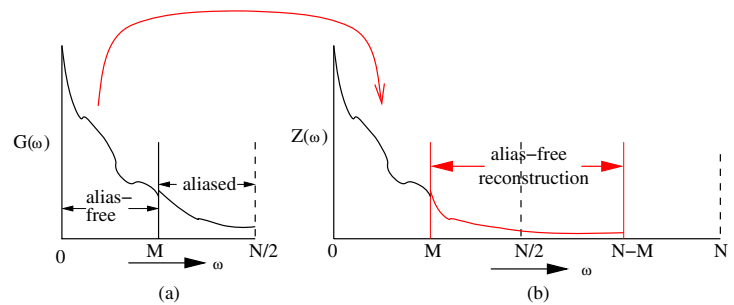

Fig. 2. Illustration of (a) partially aliased spectrum of the LR sequence $g(n)$, and (b) spectrum of the HR sequence $z(n)$ to be estimated. Note that only half of the spectrum is shown due to conjugate symmetry. 
length $2 N$ to be estimated. The alias-free interpolation method should recover the frequency components in the region $M \leq \omega \leq N-M$ in $Z(\omega)$ as shown in Figure 2 (b). From the figure, note that we have

$$
\begin{gathered}
Z(i)=q G(i) \text { for } 0 \leq i<M \\
Z(i)=0 \text { for } N-M<i \leq N
\end{gathered}
$$

and using the property of aliasing (wrapping around of frequencies)

$$
Z(i)+Z(N-i)=q G(i) \text { for } M \leq i \leq N / 2
$$

Hence the alias-free reconstruction of the high resolution signal involves recovering the spectrum $Z(\omega)$ given equations (6] 8 ). Clearly, this cannot be done without additional constraints. Note that one needs the scale factor $q$ (equal to 2 in this study) to satisfy the energy relationship (Parseval's theorem). In order to recover $z(n)$, we need state the following theorem.

Theorem 2: Given a discrete-time partially aliased 1- D signal $g \in R^{N}$, and two distinct spectral intervals $\Omega_{f}=\{0 \leq \omega<M\}$ and $\Omega_{a}=\{M \leq \omega \leq N / 2\}$ and another discrete-time signal $z \in R^{2 N}$ satisfying Nyquist criterion with three distinct spectral intervals $\Omega_{f}{ }^{\prime}=\Omega_{f}, \Omega_{a}{ }^{\prime}=\{M \leq \omega \leq N-M\}$ and $\Omega_{0}{ }^{\prime}=$ $\{N-M<\omega \leq N\}$, under conditions very similar to those defined in theorem 1, $z$ can be recovered exactly from $g$ as a solution to the $l_{1}$ minimization problem

$$
\min _{z} \sum_{n=0}^{2 N-1}|z(n)|
$$

subject to the constraints

$$
\begin{gathered}
Z(\omega)=2 G(\omega) \quad \forall \omega \in \Omega_{f^{\prime}} \\
Z(\omega)+Z(N-\omega)=2 G(\omega) \quad \forall \omega \in \Omega_{a} \\
Z(\omega)=0 \quad \forall \omega \in \Omega_{0}{ }^{\prime}
\end{gathered}
$$

One can follow arguments similar to those in [10] except that the partitions are deterministic and hence it will lead to different values of the parameters $\alpha$ and $\zeta$. It may be noted that the partitions $\Omega_{f}$ and $\Omega_{a}$ correspond to the alias-free and the aliased components of the low resolution signal $g$, respectively. Since the partition is known, it implicitly means that we know the extent of aliasing in the observation. Furthermore, the above theorem assumes that the signal consists of a limited number of Kronecker deltas. Extending the theorem to deal with piece-wise constant signal, and also on the $2-D$ lattice, we realize that we should minimize the expression

$$
\min _{z} \sum \sum\left(\left|z_{x}\right|+\left|z_{y}\right|\right)
$$

instead of equation (9) to recover the high resolution image $z$. (The multiplication factor $q$ in equations (10) and (11) should be replaced by $q^{2}=4$ due to the 
extension to $2-D$.) It may be noted that if $z$ is, indeed, piecewise constant then it cannot ideally be band limited, and hence the partition $\Omega_{f}$ will not be completely free from aliasing.

Now we look at the issue of the choice of the value of $M$ for alias removal. It is assumed in theorem 2 , that $M$ is known. This is tantamount to assuming the highest frequency component present in $z$ is known apriori. However, one would not know $M$ in practical super-resolution applications. We suggest that one solves the problem for different values of $M$ and then compare the results. However, as the value of $M$ is lowered from $N / 2$ toward 0 , the cardinality of the set $\Omega_{f}$ reduces and the reconstruction would be progressively more unreliable. It also leads to the following observation that one cannot use an interpolation factor $q$ greater than 2 as this would mean $M=0$, implying a several fold aliasing when $\Omega_{f}=\{\emptyset\}$ and hence reconstruction would be very unreliable.

\section{Computational Method}

Theorem 2 provides a theoretical basis for obtaining the alias-free interpolated image $z$. We now provide the computational tool to solve this. We obtain the solution to the above optimization problem using linear programming (LP). The objective function for the LP problem is the total variation cost as given in equation (13). The equality constraints are obtained using equations (10), (11) and (12). The equality constraints corresponding to equation (10) can be written in the form

$$
T_{f} \bar{z}=\bar{G}_{f}
$$

where $T$ is the $2 N \times 2 N$ DFT matrix with elements $T(m, n)=\left[e^{-j \pi / N}\right]^{m n}$ and $T_{f}$ represents the top $M$ rows of $T$. Thus $T_{f}$ is an $M \times 2 N$ matrix. Similarly $\bar{G}$ is the DFT of the observation $q g(n)$ and $\bar{G}_{f}$ corresponds to the top $M$ elements of $\bar{G}$. The equality constraints corresponding to equation (11) can be written as

$$
T_{a} \bar{z}=\bar{G}_{a}
$$

where $T_{a}$ is an $(N / 2-M+1) \times 2 N$ matrix whose each row is obtained by summing the corresponding two rows of the DFT matrix $T_{f}$ as per the indices shown in equation (8). $\bar{G}_{a}$ corresponds to the spectral components $(M+1)$ to $(N / 2-M)$ in $\bar{G}$. Similarly equation (12) can be written as a linear equality

$$
T_{0} \bar{z}=\overline{0}
$$

where $T_{0}$ consists of the $(N-M+1)$ to $N$ rows of the DFT matrix $T$ and $\overline{0}$ is a null vector. All the above three linear equations can now be compactly written as

$$
\left[\begin{array}{l}
T_{f} \\
T_{a} \\
T_{0}
\end{array}\right] \bar{z}=\left[\begin{array}{l}
\bar{G} \\
\overline{0}
\end{array}\right]
$$

which is of the form $C \bar{z}=\bar{d}$. We also know that $z(n) \geq 0 \forall n$ as $z(n)$ corresponds to an image. Also note that the above equation is meant for the first half of the 
spectrum. One would get an equivalent constraint for the other half based on the conjugate symmetry. Hence equations (9) and (17) constitute a standard LP problem. We have explained the problem with respect a $1-D$ signal and it should be suitably changed to handle $2-D$ images.

To solve the $l_{1}$ minimization using LP equation (13) should be written as

$$
\min _{z} \sum \sum\left(\left(z_{x}^{+}+z_{x}^{-}\right)+\left(z_{y}^{+}+z_{y}^{-}\right)\right)
$$

where $z_{x}^{+}=\max \left(z_{x}, 0\right)$ and $z_{x}^{-}=-\min \left(z_{x}, 0\right)$, etc. Unfortunately, this increases the dimensionality of the unknown variables by five fold, increasing the computation significantly. Further the constraint equations involve complex numbers when the relationships have to be split into real and imaginary parts separately, increasing the computation further. We generate the appropriate constraint matrix and solve using the linprog routine in Matlab. But, even for a $128 \times 128$ image, the computational resources required are very large. Unfortunately, Matlab fails to allocate the necessary memory even for a small sized image. A typical option in LP is to utilize the possible sparseness properties of $C$ matrix in equation (17). Unfortunately again, $C$ does not have any sparseness as the DFT matrix $T$ is not a sparse one. So we solve it as a sequential 1-D problem taking first the rows and then the columns. Hence the results obtained by this method for images in this paper are all sub-optimal.

\section{Experimental Results}

In this section we present the results of alias-free interpolation obtained using the proposed approach. All the LR images are of size $64 \times 64$. All the results shown in this section are for interpolation factor of 2 for the reason described in section 4. Since the amount of aliasing $M$ is not known, we show results for various choices of $M$.

First we show the applicability of the proposed method on a simulated $1-D$ data. Figure 3 (a) shows a low resolution rectangular pulse train and the corresponding spectrum is shown in Figure 3 (b). The signal shown in Figure 3 (a) is superimposed with three high frequency components corresponding to the normalized frequencies $35 / 64,36 / 64$ and $37 / 64$ to obtain the signal shown in Figure 3(c). Clearly, these three spectral components are aliased ones. Figure 3 (d) shows the spectrum of the aliased signal. One cannot find that the signal is aliased either from Figures 3 (c) or 3 (d). Figure 3 (e) shows the interpolated signal using the proposed method and its spectrum is shown in Figure 3 (f). One can see that there are spectral components at locations beyond the normalized frequency $32 / 128$. These components match quite well with the introduced high frequency components. We have used $M=26$ in this example. To further see the gain arising out of the proposed method, one can note that the spectrum of the rectangular pulse train (without the additional high frequency components) shown in Figure 3 (b) compares very favorably with the spectrum of the interpolated signal till the normalized frequency of $32 / 128$. On 


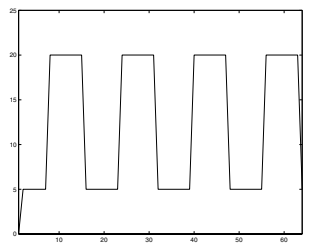

(a)

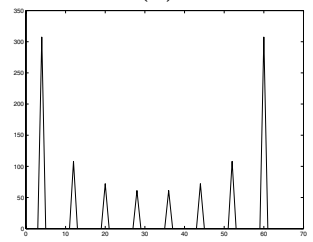

(b)

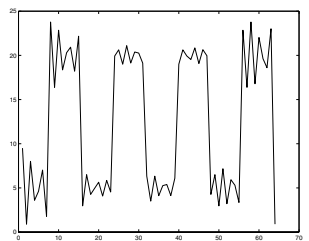

(c)

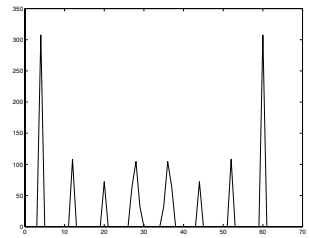

(d)

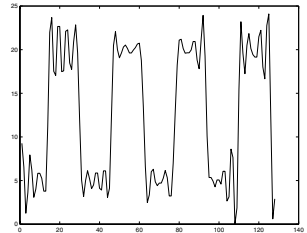

(e)

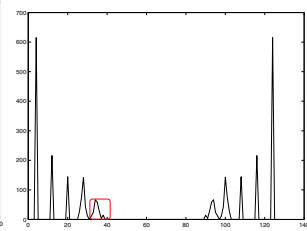

(f)

Fig. 3. Demonstration of the proposed approach for a $1-D$ signal: (a) alias-free LR signal, (b) spectrum of (a), (c) aliased LR signal, (d) spectrum of (c), (e) interpolated signal using the proposed approach and (f) spectrum of (e)

comparing the interpolated signal in Figure 3 (e) with the low resolution signal in Figure 3 (c), one can clearly see that Figure 3 (e) cannot be obtained by the linear or cubic interpolation of the original signal. (see the highlighted spectral components in Figure 3 (f)). This confirms the utility of the proposed method. Further, to illustrate the capability of our method we compare the results of our alias free interpolation with spline interpolation in Figure 4. Figure 4(a) shows a low resolution rectangular pulse train and the corresponding spectrum is shown in Figure 4 (b). The spline interpolated result and the corresponding spectrum are shown in Figures 4 (c) and (d) respectively. The alias free interpolated signal and its spectrum are shown in Figures 4 (e) and (f), respectively. As can be observed, the alias free interpolated signal is almost free from overshoot and ripples as compared to the spline interpolated one.

Figure 5 (a) shows a partially aliased low resolution Lena image of size $64 \times 64$. Figure 5 (b) shows the bicubic interpolated image for comparison to the proposed method. Figures 5 (c-e) show the alias-free interpolation results obtained using the sub-optimal linear programming method. Figure 5 (c) corresponds to the result where 10\% ( $M=29$ ), additional high frequency components are generated. Here we assume that the aliasing in the LR image is small, only $10 \%$ of the entire spectrum. If we assume that the aliasing in the LR observation is about $20 \%$, the corresponding alias-free interpolated image is shown in Figure 5 (d). This corresponds to the choice of $M=26$. Figure 5 (e) shows the alias-free interpolated image where we attempt to generate $30 \%$ additional high frequency components assuming that $30 \%$ of the spectrum of the LR image is aliased. As can be observed from Figures 5 (c-e), there is a gradual reduction in the quality of the reconstructed image as the aliasing in the LR image is assumed to have increased from $10 \%$ to $30 \%$. This is due to the fact that only a smaller subset of spectral components are known exactly. In comparison to 


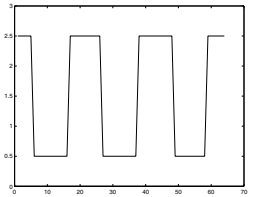

(a)

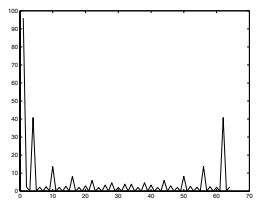

(b)

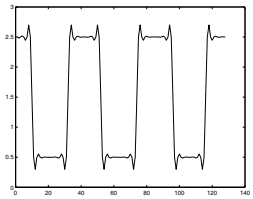

(c)

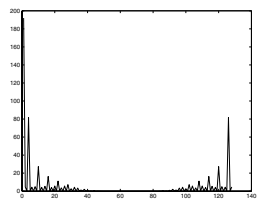

(d)

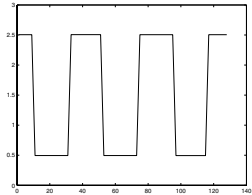

(e)

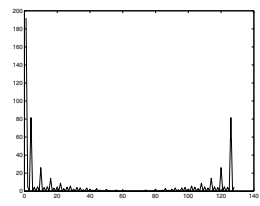

(f)

Fig. 4. Demonstration of the proposed approach for another $1-D$ signal: (a) LR signal, (b) spectrum of (a), (c) spline interpolated signal, (d) spectrum of (c), (e) interpolated signal using the proposed approach and (f) spectrum of (e)

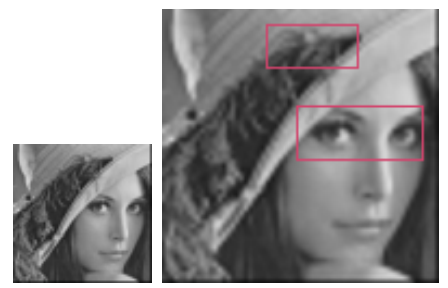

(a)

(b)

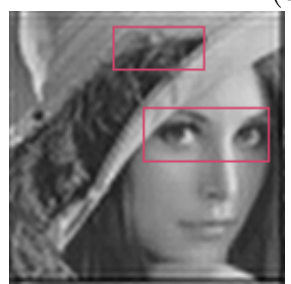

(c)

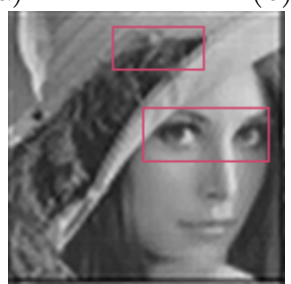

(d)

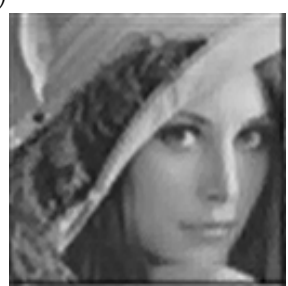

(e)

Fig. 5. (a) A low resolution Lena image, (b) bicubic interpolated image. Interpolated images using the proposed approach generating additional (c) $10 \%$, (d) $20 \%$ and (e) $30 \%$ high frequency components.

the bicubic interpolated image, the result using the proposed approach is much sharper. Observe the eyes, hair strands, etc. in Figure 5(d). Some of the regions are highlighted in the figure. We have observed that the reconstruction becomes poor when the aliasing present in the LR image is assumed to be more than 20 to $30 \%$. Now we perform the experiments on a severely aliased randomly textured image. The purpose of this experiment is to demonstrate that one does not get any improvement during interpolation if the signal is highly aliased. The LR observation is shown Figure 6 (a). The interpolated images using the proposed 


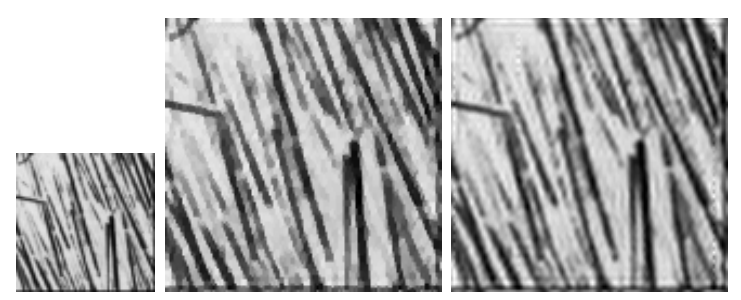

(a)

(b)

(c)

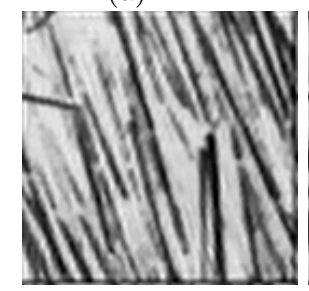

(d)

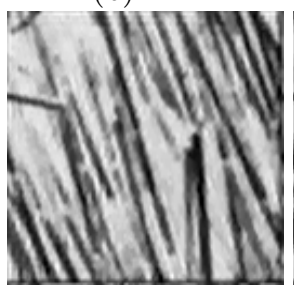

(e)

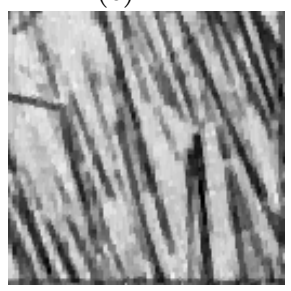

(f)

Fig. 6. (a) A severely aliased low resolution texture image, (b) bicubic interpolated image. Interpolated images using the proposed approach generating (c) $10 \%$, (d) $20 \%$ and (e) $30 \%$ high frequency components, (f) Interpolated image when the LR image is fully aliased.

approach are shown in Figures 6 (c-e) assuming 10\%, 20\% and 30\% aliasing, respectively, in the given $\mathrm{LR}$ image. As the aliasing present in the LR image is very high, the proposed method does not give a significant edge over bicubic interpolation as can be observed from Figures [6 (c-e). Now we assume that the entire spectrum is aliased, ie, $\Omega_{f}=\{\emptyset\}$ in theorem $2(\mathrm{M}=1)$. Figure [6 (f) shows the corresponding interpolated result. We observe that the reconstruction is quite inferior as we do not have any of the spectral components known exactly.

\section{Conclusion}

In this paper we have presented a method for alias-free interpolation from a partially aliased low resolution image. We have provided a theoretical basis on how an alias-free upsampling can be achieved. In order to interpolate the given LR image we generate the exact additional high frequency components assuming a knowledge of the nature of aliasing in the spectrum of the LR observation and assuming a piecewise constant intensity image. The alias-free interpolation is achieved by solving the $l_{1}$ optimization. A sub-optimal computational procedure using linear programming is also presented.

\section{References}

1. Chaudhuri, S., Joshi, M.V.: Motion-Free Super-Resolution. Springer (2005)

2. Tsai, R.Y., Huang, T.S.: Multiframe Image Restoration and Registration. In: Advances in Computer Vision and Image Processsing. JAI Press Inc. (1984) 317-339 
3. Irani, M., Peleg, S.: Improving Resolution by Image Registration. CVGIP: Graphical Models and Image Processing 53 (1991) 231-239

4. Schultz, R.R., Stevenson, R.L.: A Bayesian Approach to Image Expansion for Improved Definition. IEEE Trans. on Image Processing 3 (1994) 233-242

5. Elad, M., Feuer, A.: Restoration of a Single Superresolution Image from Several Blurred, Noisy and Undersampled Measured Images. IEEE Trans. on Image Processing 6 (1997) 1646-1658

6. Nguyen, N., Milanfar, P., Golub, G.: A Computationally Efficient Super-resolution Reconstruction Algorithm. IEEE Trans. Image Processing 10 (2001) 573-583

7. Lin, Z., Shum, H.Y.: Fundamental Limits of Reconstruction-Based SuperResolution Algorithms under Local Translation. IEEE Trans. on Pattern Analysis and Machine Intelligence 26 (2004) 83-97

8. Shahram, M., Milanfar, P.: Imaging Below the Diffraction Limit: A Statistical Analysis. IEEE Trans. on Image Processing 13 (2004) 677-689

9. Rajan, D., Chaudhuri, S.: Generalized Interpolation and its Applications in SuperResolution imaging. Image and Vision Computing 19 (2001) 957-969

10. Candes, E., Romberg, J., Tao, T.: Robust Uncertainty Principles: Exact Signal Reconstruction from Highly Incomplete Frequency Information (2004) http: //www.acm.caltech.edu/ emmanuel/publications.html.

11. Donoho, D.L., Elad, M.: Maximal sparsity Representation via $l_{1}$ Minimization. the Proc. Nat. Aca. Sci. 100 (2003) 2197-2202

12. Bertalmio, M., G. Sapiro, V.C., Ballester, C.: Image Inpainting. In: Proc. SIGGRAPH, New Orleans, USA (2000)

13. Chan, T., Kang, S.H.: Error Analysis for Image Inpainting. UCLA CAM report 04-72 (2004)

14. Levy, S., Fullagar, P.K.: Reconstruction of a sparse spike train from a portion of its spectrum and application to high-resolution deconvolution. GEOPHYSICS 46 (1981) 1235-1243

15. Santosa, F., Symes, W.W.: Linear inversion of band-limited reflection seismograms. SIAM J. Sci. Statist. Comput. 7 (1986) 1307-1330

16. Guichard, F., Malgouyres, F.: Total Variation Based Interpolation. In: Proc. European Signal Processing Conference. (1998) 1741-1744

17. Malgouyres, F., Guichard, F.: Edge Direction Preserving Image Zooming: A Mathematical and Numerical Analysis. SIAM J. Numerical Analysis 39 (2001) 1-37

18. Rudin, L., Osher, S., Fatemi, E.: Nonlinear total variation based noise removal algorithms. PHYSICA D 60 (1992) 259-268 